Article

\title{
Adequate Knowledge of Stroke Symptoms, Risk Factors, and Necessary Actions in the General Population of Southern Poland
}

\author{
Ewa Krzystanek ${ }^{1, *(1)}$, Agnieszka Krzak-Kubica ${ }^{2}$, Maciej Świat ${ }^{3}$, Weronika Galus ${ }^{1}$ \\ and Justyna Gawryluk ${ }^{1}$ \\ 1 Department of Neurology, Faculty of Medical Sciences in Katowice, Medical University of Silesia, Katowice, \\ ul. Medykow 14, 40-752 Katowice, Poland; weroni7@gmail.com (W.G.); jgawryluk@sum.edu.pl (J.G.) \\ 2 Department of Neurology, University Central Hospital, Katowice, ul. Medykow 14, 40-752 Katowice, Poland; \\ agukrzak@gmail.com \\ 3 Jan Dlugosz University, ul. Waszyngtona 4/8, 42-200 Czestochowa, Poland; maciejswiat@gmail.com \\ * Correspondence: ekrzystanek@sum.edu.pl
}

Received: 3 November 2020; Accepted: 8 December 2020; Published: 18 December 2020

\begin{abstract}
Background and objectives: Stroke is the third most common cause of mortality in developed countries and the primary cause of neurological disability in adults. Recombinant tissue plasminogen activator administered intravenously within $4.5 \mathrm{~h}$ from the onset of symptoms constitutes a gold standard in the treatment of acute ischemic stroke. Prompt hospital admission is the prerequisite of effective thrombolysis. Therefore, stroke awareness in the general population is the key factor in timely recognition of the acute stroke victims and determines proper actions. Therefore, the purpose of this study was to determine the awareness of stroke in the general population of the Silesian voivodeship, the most populated region of Poland. We assessed also the "adequate knowledge of stroke", a combined measure of the optimal level of stroke awareness, as a prerequisite for effective stroke management, and aimed to identify most impacting factor for adequate stoke knowledge, to help shaping education strategies. Materials and Methods: A proprietary anonymous questionnaire consisting of 15 items related to stroke was used in this research. A total of 1134 individuals were surveyed. Additionally to the knowledge of individual aspects of stroke, we assessed "adequate knowledge of stroke", which was combined measure of risk factors, symptoms, and actions in the case of acute stroke. Results: The accurate definition of stroke was selected by 834 participants $(73.5 \%)$. The vast majority of them indicated that a stroke is an emergency $(92.8 \%)$ and medical assistance is required $(97.5 \%)$. However, $42.4 \%$ of respondents did not know any specific symptom of stroke and only $38.6 \%$ participants were able to list two or more risk factors, which resulted in only $36.3 \%$ of individuals with adequate knowledge of stroke. Education duration, previous occurrence of stroke in relatives or friends, gender and place of residence were identified as independent predictors of adequate knowledge of stroke. Conclusions: 1 . Knowledge of stroke in the population of southern Poland is low and may be considered insufficient to address the needs of timely management in the action chain. 2. Previous occurrence of stroke in the relatives or friends is the most impacting factor for adequate knowledge of stroke.
\end{abstract}

Keywords: stroke; stroke risk factors; stroke symptoms; stroke awareness; stroke knowledge; health education

\section{Introduction}

Stroke is the third most common cause of mortality in developed countries and the primary cause of neurological disability in adults $[1,2]$. Due to the ageing of the worldwide population, it is estimated 
that in the near future, stroke will be the primary cause of lost years of life [3]. Every year, stroke affects 60,000 people in Poland [4], of which up to $40 \%$ die within the first month [5], and one-third die within the first year afterwards [2].

Efficacy of acute stroke treatment is time-dependent. Recombinant tissue plasminogen activator (t-PA) for acute ischemic stroke is currently a gold standard in stroke management, but requires administration within $4.5 \mathrm{~h}$ from the onset of symptoms [6]. Mechanical thrombectomy, indicated in the acute treatment of ischemic strokes caused by an emergent large-vessel occlusion (ELVO), may be performed within $6 \mathrm{~h}$ in case of anterior circulation infarcts. Studies show, that interventions are more beneficial, if performed early. Delayed arrival to the hospital is, therefore, the primary obstacle for the effective treatment [7-9]. The main reason for this delay is the lack of public awareness of stroke, its symptoms, risk factors or appropriate behaviors (e.g., immediate emergency call). This problem has been identified in many countries, but in Poland, only two studies were published [10-18]. Better understanding of perceived risk factors and warning signs of stroke would facilitate health interventions aimed at reducing stroke-associated morbidity and mortality.

Therefore, the purpose of this study was to determine the awareness of stroke in the general population of the Silesian voivodeship, the most populated region of Poland. We assessed also the "adequate knowledge of stroke", a combined measure of the optimal level of stroke awareness, as a prerequisite for effective stroke management, and aimed to identify most impacting factor for adequate stoke knowledge, to help shaping education strategies.

\section{Materials and Methods}

This was a cross-sectional sampling investigation study of a survey of adult inhabitants from the Polish region of Silesia, with the population around 4.5 million. This survey was carried out by medical students from the Students' Scientific Organization of the Department of Neurology (at the Medical University of Silesia, Katowice, Poland) and the authors over a period of 2 years (2011-2012). The sample structure, to be most representative for the population of interest, was defined based on demographic analysis published in 2010 by Statistical Office in Katowice, Poland [19]. The participants were recruited from several places: private homes, waiting rooms of various clinics, cultural centers, and various artistic and cultural events as well as Third Age Universities. The subject and objectives of the study were clarified prior to the interview to each of participants, and oral consent was obtained. Additionally to the general population studied sample, we questioned medical students from the first and sixth year of the medical school, but the collected data are not presented here, as the purpose and objectives were different and may bias the results.

We study used an anonymous, original, 15-item questionnaire to ascertain participant knowledge of stroke. The questionnaire was developed based on FAST test criteria (F-face, A-arm, S-speech, T-time) and available sociological tests used in population-based studies of stroke. The questionnaire consisted of two sections gathering personal data and stroke knowledge.

In the first part, sociodemographic data such as age, gender, level of education, profession, and place of residence (urban vs. rural), as well as previous occurrence of stroke in the family or close friends were collected. For the purpose of the analysis, participants were categorized by age ( $<45$ years and $\geq 45$ years of age) and by level of education ( $<10$ years and $\geq 10$ years of education).

The second part consisted of three open-ended and twelve closed-ended questions pertaining to the definition of stroke, risk factors, symptoms of stroke, action taken in case of stroke, or healthy behaviors to reduce the risk of stroke, as well as information sources, which an individual would use to inform himself or herself. Open-ended questions are considered to better represent actual knowledge of respondents; therefore, in the questionnaire they were asked before the close-ended questions, to avoid prompting the answers. A copy of the questionnaire may be requested from the corresponding author.

For the purpose of the analysis, all answers to the open-ended questions were grouped into specific categories, in order to represent responses typical for the respective question or topic. In case of 
ambiguity, trained neurologist with more than 10 years of experience in stroke management categorized the responses to best match the defined criteria. Answers directing to several different categories, or clearly out of the scope of questionnaire were grouped in the "other" category. The response to the question about the definition of stroke was considered correct, if in the close-ended question of multiple choice the participant selected either "a condition when a blood vessel within the head bursts and bleeds into and around the brain", or "a condition when a blood clot blocks the flow of blood in the artery in the brain", or both. If any other answer was selected, the whole response was considered incorrect. Responses "I don't know" or missing, e.g., if the participant did not provide any answer to single question or left the space blank, were combined together as incorrect.

Adequate knowledge of stroke was defined based on previously proposed criteria by Ramirez-Moreno et al. [20,21], with minor modifications adopted by consensus. The participants were considered as presenting "adequate knowledge of stroke", if they correctly named all three:

- at least 1 of 5 major symptoms: consciousness disturbances, speech disturbances, face or mouth asymmetry, paresis or paralysis, or sensory symptoms,

- at least 1 of 11 main risk factors: high blood pressure, smoking, physical inactivity, obesity, alcohol abuse, poor diet/nutrition, hypercholesterolemia, diabetes, cardiovascular diseases, old age, genetic/family history

- call to emergency as a proper action in the case of the acute stroke.

Statistical analysis was performed with use of SYSTAT 12 software (SPSS science, Chicago, IL, USA). Summary data were presented as numbers or proportions of studied group or feature. To analyze differences between the studied groups, chi-square test or the Fisher exact test for categorical data and t-test or Mann-Whitney U test for continuous data were used. For ordinal variables and those with more than 2 categories, we used the Cochran-Mantel-Haenszel test to analyze trends. A univariate analysis was performed to compare "adequate knowledge of stroke" with demographic and socioeconomic information, as well as previous experience with the disease. Binary logistic regression model was constructed to identify independent factors that may explain the "adequate knowledge of stroke" (dependent variable). Variables were selected using stepwise backward elimination, using $p$ greater than 0.15 as exclusion and $p$ less than 0.05 as inclusion criteria. A $p$ value below 0.05 was considered significant.

\section{Results}

A total of 1134 questionnaires were collected, of which 752 were completed by women $(66.3 \%$ of all respondents). The mean age $( \pm \mathrm{SD})$ of the participants was $42.6 \pm 15.3$ years (ranging between 23-79 years) and the mean age of men was higher than women $(p=0.001)$. City inhabitants under the age of 45 years, with at least 10 years of school education constituted the majority of respondents and proportion of women with higher education level was greater than men $(p<0.001)$. Among the professional groups, the most represented were white-collar workers and retired professionals/pensioners. The most common sources of stroke-related information were television and the press. Less than one-fifth of the participants received stroke-related information from a doctor and $6.4 \%$ of the participants have never come across any stroke-related information. Detailed demographic characteristics and the source of stroke-related information are presented in the Table 1. 
Table 1. Group characteristics and source of information about stroke.

\begin{tabular}{|c|c|c|c|c|c|c|c|c|}
\hline \multirow{2}{*}{ All } & & \multicolumn{2}{|c|}{ Total $(n \%)$} & \multicolumn{2}{|c|}{$\operatorname{Men}(n \%)$} & \multicolumn{2}{|c|}{ Women $(n \%)$} & \multirow{2}{*}{$p$} \\
\hline & & 1134 & $100 \%$ & 382 & $33.7 \%$ & 752 & $66.3 \%$ & \\
\hline \multirow[t]{2}{*}{ Age, years } & mean $( \pm S D)$ & 42.6 & (15.3) & 44.7 & $(15.6)$ & 41.5 & (15.1) & 0.001 \\
\hline & $\begin{array}{l}\text { median } \\
(\min -\max )\end{array}$ & 37 & $(23-79)$ & 39 & $(24-75)$ & 36 & $(23-79)$ & \\
\hline \multirow[t]{2}{*}{ Age group } & $<45$ years & 703 & $62.0 \%$ & 215 & $30.6 \%$ & 488 & $69.4 \%$ & 0.005 \\
\hline & $\geq 45$ years & 431 & $38.0 \%$ & 167 & $38.7 \%$ & 264 & $61.3 \%$ & \\
\hline \multirow{4}{*}{$\begin{array}{l}\text { Place of } \\
\text { residence } \\
\text { Education } \\
\text { duration }\end{array}$} & urban & 735 & $64.8 \%$ & 239 & $32.5 \%$ & 496 & $67.5 \%$ & 0.258 \\
\hline & rural & 399 & $35.2 \%$ & 143 & $35.8 \%$ & 256 & $64.2 \%$ & \\
\hline & $<10$ years & 281 & $24.8 \%$ & 131 & $46.6 \%$ & 150 & $53.4 \%$ & $<0.001$ \\
\hline & $\geq 10$ years & 853 & $75.2 \%$ & 251 & $29.4 \%$ & 602 & $70.6 \%$ & \\
\hline \multirow[t]{6}{*}{ Profession } & $\begin{array}{l}\text { white-collar } \\
\text { worker }\end{array}$ & 452 & $39.9 \%$ & 116 & $25.7 \%$ & 336 & $74.3 \%$ & $<0.001$ \\
\hline & retired & 273 & $24.1 \%$ & 109 & $39.9 \%$ & 164 & $60.1 \%$ & \\
\hline & $\begin{array}{l}\text { manual } \\
\text { worker }\end{array}$ & 225 & $19.8 \%$ & 117 & $52.0 \%$ & 108 & $48.0 \%$ & \\
\hline & unemployed & 77 & $6.8 \%$ & 15 & $19.5 \%$ & 62 & $80.5 \%$ & \\
\hline & $\begin{array}{l}\text { health care } \\
\text { worker }\end{array}$ & 74 & $6.5 \%$ & 9 & $12.2 \%$ & 65 & $87.8 \%$ & \\
\hline & $\begin{array}{l}\text { company } \\
\text { owner }\end{array}$ & 33 & $2.9 \%$ & 16 & $48.5 \%$ & 17 & $51.5 \%$ & \\
\hline \multirow{2}{*}{\multicolumn{2}{|c|}{$\begin{array}{l}\text { Stroke in relatives or friends } \\
\text { Source of stroke information }\end{array}$}} & 327 & $28.8 \%$ & 87 & $26.6 \%$ & 240 & $73.4 \%$ & \\
\hline & & & & & & & & \\
\hline \multicolumn{2}{|c|}{ TV } & 666 & $58.7 \%$ & & & & & \\
\hline \multicolumn{2}{|l|}{ radio } & 271 & $23.9 \%$ & & & & & \\
\hline \multicolumn{2}{|l|}{ press } & 427 & $37.7 \%$ & & & & & \\
\hline \multicolumn{2}{|c|}{ internet } & 242 & $21.3 \%$ & & & & & \\
\hline \multicolumn{2}{|c|}{ doctor's office } & 204 & $18.0 \%$ & & & & & \\
\hline \multicolumn{2}{|c|}{ other } & 278 & $24.5 \%$ & & & & & \\
\hline \multicolumn{2}{|l|}{ none } & 73 & $6.4 \%$ & & & & & \\
\hline
\end{tabular}

Correct definition of stroke was selected as an answer to a close-ended question by 834 (73.5\%) participants and place of residence, education duration, or previous stroke in relatives or friends determined differences between subgroups (Table 2). The vast majority of participants indicated that a stroke is an emergency, which requires hospitalization ( $92.8 \%$ and $97.5 \%$, respectively). Stroke was more often considered an emergency by women than men $(p=0.003)$ and individuals with higher education level ( $p=0.01$ ), but the need for hospitalization was identified equally often in all groups. A call to emergency services, as a response to a close-ended question, considered the only correct action in case of acute stroke, was selected by $82.6 \%$ participants, where those with higher education level and previous experience of stroke in relatives or friends were more likely to call an ambulance $(p=0.017$ and $p<0.001$, respectively) (Table 2$)$.

Suspected risk factors provided by participants to an open-ended question were sorted in 12 main categories and "other" category for unclassifiable responses (Table 3). $860(76.8 \%)$ participants were able to name at least one risk factor, while $438(38.6 \%)$ listed two or more risk factors. The most frequently mentioned risk factor was old age (55.3\%), which was almost twice as frequent as high blood pressure $(29.2 \%)$ and almost five times more frequent than stress (13.1\%). Hypercholesterolemia, diabetes, and genetic/family history were listed by less than $3 \%$ of all the respondents. Gender, place of residence and education level and previous stroke in relatives or friends were associated with differences in proportions of identified risk factors between groups. Women, better educated individuals, those living in the city and who had previous contact with stroke listed more risk factors than their counterparts. $274(24.2 \%)$ participants did not provide any response, or selected "I don't know". 
Table 2. Correct stroke definition and proper actions required in case of stroke reported by participants grouped by gender, age group, place of residence, education level, and profession.

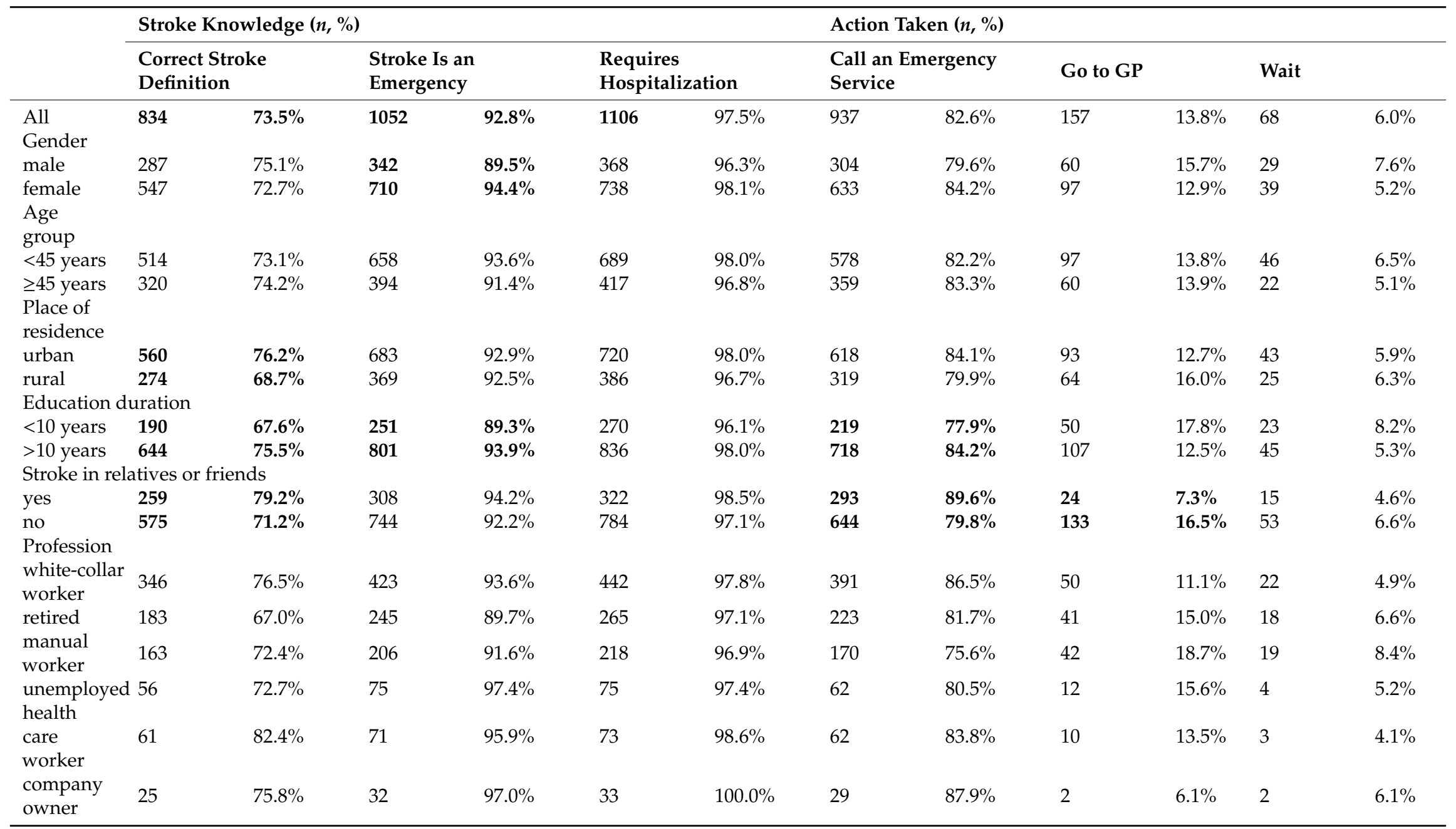

Note: Between-groups comparisons performed with chi-squared test, bold indicates $p<0.05$. Proportions in the Profession category were not analyzed. Responses "Go to GP" and "Wait" were not analyzed separately, as they were the part of multiple choice question pertaining to an emergency action. 
Table 3. Stroke risk factors provided by participants in response to an open-ended question grouped by gender, age group, place of residence, education level and profession.

\begin{tabular}{|c|c|c|c|c|c|c|c|c|c|c|c|c|c|c|}
\hline & $\begin{array}{l}\text { High } \\
\text { Blood } \\
\text { Pressure }\end{array}$ & Smoking & $\begin{array}{l}\text { Physical } \\
\text { Inactivity }\end{array}$ & Obesity & $\begin{array}{l}\text { Alcohol } \\
\text { Abuse }\end{array}$ & $\begin{array}{l}\text { Poor } \\
\text { Diet/Nutrition }\end{array}$ & Hypercholesterolemia & Diabetes & $\begin{array}{l}\text { Cardiovascular } \\
\text { Diseases }\end{array}$ & $\begin{array}{l}\text { Old } \\
\text { Age }\end{array}$ & Stress & $\begin{array}{l}\text { Genetic/Family } \\
\text { History }\end{array}$ & Other & $\begin{array}{l}\text { Don't } \\
\text { Know/No } \\
\text { Response }\end{array}$ \\
\hline \multicolumn{15}{|l|}{ All } \\
\hline$n$ & 331 & 122 & 58 & 34 & 78 & 83 & 25 & 32 & 98 & 627 & 148 & 26 & 38 & 274 \\
\hline$\%$ & 29.2 & 10.8 & 5.1 & 3.0 & 6.9 & 7.3 & 2.2 & 2.8 & 8.6 & 55.3 & 13.1 & 2.3 & 3.4 & 24.2 \\
\hline \multicolumn{15}{|l|}{ Gender } \\
\hline $\begin{array}{l}\text { female, } \\
n\end{array}$ & 249 & 85 & 37 & 24 & 51 & 62 & 22 & 26 & 71 & 412 & 97 & 16 & 25 & 178 \\
\hline$\%$ & 33.1 & 11.3 & 4.9 & 3.2 & 6.8 & 8.2 & 2.9 & 3.5 & 9.4 & 54.8 & 12.9 & 2.1 & 3.3 & 23.7 \\
\hline male, $n$ & 82 & 37 & 21 & 10 & 27 & 21 & 3 & 6 & 27 & 215 & 51 & 10 & 13 & 96 \\
\hline$\%$ & 21.5 & 9.7 & 5.5 & 2.6 & 7.1 & 5.5 & 0.8 & 1.6 & 7.1 & 56.3 & 13.4 & 2.6 & 3.4 & 25.1 \\
\hline \multicolumn{15}{|l|}{$\begin{array}{l}\text { Age } \\
\text { group }\end{array}$} \\
\hline $\begin{array}{l}<45 \\
\text { years, } n\end{array}$ & 200 & 79 & 37 & 23 & 42 & 50 & 11 & 17 & 69 & 392 & 81 & 9 & 23 & 181 \\
\hline$\%$ & 28.4 & 11.2 & 5.3 & 3.3 & 6.0 & 7.1 & 1.6 & 2.4 & 9.8 & 55.8 & 11.5 & 1.3 & 3.3 & 25.7 \\
\hline $\begin{array}{l}\geq 45 \\
\text { years, } n\end{array}$ & 131 & 43 & 21 & 11 & 36 & 33 & 14 & 15 & 29 & 235 & 67 & 17 & 15 & 93 \\
\hline$\%$ & 30.4 & 10.0 & 4.9 & 2.6 & 8.4 & 7.7 & 3.2 & 3.5 & 6.7 & 54.5 & 15.5 & 3.9 & 3.5 & 21.6 \\
\hline \multicolumn{15}{|c|}{ Place of residence * } \\
\hline urban, $n$ & 238 & 92 & 46 & 25 & 60 & 57 & 21 & 25 & 72 & 401 & 109 & 22 & 33 & 159 \\
\hline$\%$ & 32.4 & 12.5 & 6.3 & 3.4 & 8.2 & 7.8 & 2.9 & 3.4 & 9.8 & 54.6 & 14.8 & 3.0 & 4.5 & 21.6 \\
\hline rural, $n$ & 93 & 30 & 12 & 9 & 18 & 26 & 4 & 7 & 26 & 226 & 39 & 4 & 5 & 115 \\
\hline$\%$ & 23.3 & 7.5 & 3.0 & 2.3 & 4.5 & 6.5 & 1.0 & 1.8 & 6.5 & 56.6 & 9.8 & 1.0 & 1.3 & 28.8 \\
\hline \multicolumn{15}{|c|}{ Education duration * } \\
\hline $\begin{array}{l}<10 \\
\text { years, } n\end{array}$ & 66 & 20 & 6 & 8 & 13 & 14 & 3 & 2 & 16 & 138 & 30 & 4 & 7 & 86 \\
\hline$\%$ & 23.5 & 7.1 & 2.1 & 2.8 & 4.6 & 5.0 & 1.1 & 0.7 & 5.7 & 49.1 & 10.7 & 1.4 & 2.5 & 30.6 \\
\hline $\begin{array}{l}>10 \\
\text { years, } n\end{array}$ & 265 & 102 & 52 & 26 & 65 & 69 & 22 & 30 & 82 & 489 & 118 & 22 & 31 & 188 \\
\hline$\%$ & 31.1 & 12.0 & 6.1 & 3.0 & 7.6 & 8.1 & 2.6 & 3.5 & 9.6 & 57.3 & 13.8 & 2.6 & 3.6 & 22.0 \\
\hline
\end{tabular}


Table 3. Cont.

\begin{tabular}{|c|c|c|c|c|c|c|c|c|c|c|c|c|c|c|}
\hline & $\begin{array}{l}\text { High } \\
\text { Blood } \\
\text { Pressure }\end{array}$ & Smoking & $\begin{array}{l}\text { Physical } \\
\text { Inactivity }\end{array}$ & Obesity & $\begin{array}{l}\text { Alcohol } \\
\text { Abuse }\end{array}$ & $\begin{array}{l}\text { Poor } \\
\text { Diet/Nutrition }\end{array}$ & Hypercholesterolemia & Diabetes & $\begin{array}{l}\text { Cardiovascular } \\
\text { Diseases }\end{array}$ & $\begin{array}{l}\text { Old } \\
\text { Age }\end{array}$ & Stress & $\begin{array}{l}\text { Genetic/Family } \\
\text { History }\end{array}$ & Other & $\begin{array}{l}\text { Don't } \\
\text { Know/No } \\
\text { Response }\end{array}$ \\
\hline \multicolumn{15}{|c|}{ Stroke in relatives or friends * } \\
\hline yes, $n$ & 144 & 52 & 23 & 15 & 29 & 45 & 10 & 20 & 44 & 197 & 62 & 11 & 11 & 37 \\
\hline$\%$ & 44.0 & 15.9 & 7.0 & 4.6 & 8.9 & 13.8 & 3.1 & 6.1 & 13.5 & 60.2 & 19.0 & 3.4 & 3.4 & 11.3 \\
\hline no, $n$ & 187 & 70 & 35 & 19 & 49 & 38 & 15 & 12 & 54 & 430 & 86 & 15 & 27 & 237 \\
\hline$\%$ & 23.2 & 8.7 & 4.3 & 2.4 & 6.1 & 4.7 & 1.9 & 1.5 & 6.7 & 53.3 & 10.7 & 1.9 & 3.3 & 29.4 \\
\hline \multicolumn{15}{|l|}{ Profession } \\
\hline $\begin{array}{l}\text { white-collar } \\
\text { worker, } \\
n\end{array}$ & 140 & 52 & 29 & 17 & 32 & 44 & 10 & 20 & 55 & 262 & 59 & 11 & 14 & 101 \\
\hline$\%$ & 31.0 & 11.5 & 6.4 & 3.8 & 7.1 & 9.7 & 2.2 & 4.4 & 12.2 & 58.0 & 13.1 & 2.4 & 3.1 & 22.3 \\
\hline $\begin{array}{l}\text { retired, } \\
n\end{array}$ & 77 & 21 & 9 & 6 & 19 & 7 & 8 & 5 & 17 & 140 & 40 & 7 & 13 & 73 \\
\hline$\%$ & 28.2 & 7.7 & 3.3 & 2.2 & 7.0 & 2.6 & 2.9 & 1.8 & 6.2 & 51.3 & 14.7 & 2.6 & 4.8 & 26.7 \\
\hline $\begin{array}{l}\text { manual } \\
\text { worker, } \\
n\end{array}$ & 48 & 18 & 9 & 5 & 10 & 15 & 0 & 1 & 12 & 118 & 24 & 3 & 4 & 63 \\
\hline$\%$ & 21.3 & 8.0 & 4.0 & 2.2 & 4.4 & 6.7 & & 0.4 & 5.3 & 52.4 & 10.7 & 1.3 & 1.8 & 28.0 \\
\hline $\begin{array}{l}\text { unemploye } \\
n\end{array}$ & & 8 & 2 & 0 & 4 & 4 & 2 & 1 & 0 & 47 & 6 & 2 & 2 & 24 \\
\hline$\%$ & 14.3 & 10.4 & 2.6 & & 5.2 & 5.2 & 2.6 & 1.3 & & 61.0 & 7.8 & 2.6 & 2.6 & 31.2 \\
\hline $\begin{array}{l}\text { health } \\
\text { care } \\
\text { professiona } \\
n\end{array}$ & & 17 & 6 & 5 & 9 & 10 & 3 & 5 & 12 & 46 & 11 & 3 & 5 & 6 \\
\hline$\%$ & 59.5 & 23.0 & 8.1 & 6.8 & 12.2 & 13.5 & 4.1 & 6.8 & 16.2 & 62.2 & 14.9 & 4.1 & 6.8 & 8.1 \\
\hline $\begin{array}{l}\text { private } \\
\text { business, } \\
n\end{array}$ & 11 & 6 & 3 & 1 & 4 & 3 & 2 & 0 & 2 & 14 & 8 & 0 & 0 & 7 \\
\hline$\%$ & 33.3 & 18.2 & 9.1 & 3.0 & 12.1 & 9.1 & 6.1 & & 6.1 & 42.4 & 24.2 & & & 21.2 \\
\hline
\end{tabular}

Note: Between-groups comparisons performed with Cochran-Mantel-Haenszel test, ${ }^{*}$ indicates $p<0.05$. Proportions in the Profession category were not analyzed. 
Suspected symptoms of stroke provided by participants to an open-ended question were sorted in eight main categories and "other" category for unclassifiable responses (Table 4). 653 (57.6\%) participants were able to name at least one sign or symptom, while $399(35.2 \%)$ listed two or more risk factors. The most frequently listed symptoms included the consciousness disturbances $(33.4 \%)$, headaches $(26.2 \%)$ and paresis $(19.5 \%)$. The least frequently named symptom was face/mouth asymmetry (3.3\%) (part of FAST test), whereas vision disturbances or vision loss were not mentioned at all, thus not included in the table. Similarly to risk factors, gender, place of residence, education duration and previous stroke in relatives or friends were associated with differences in proportions of identified symptoms between subgroups and also here, women, better educated individuals, those living in the city and who had previous contact with stroke listed more symptoms than their counterparts. 481 (42.4\%) participants did not provide any response, or selected "I don't know".

Stroke prevention strategies were listed in a closed-ended question. The most often selected answers were healthy lifestyle, blood pressure monitoring and smoking cessation $(77.3 \%, 68 \%$, and $60 \%$, respectively). Almost $60 \%$ of respondents indicated that regular physical activity is beneficial too, whilst $23.4 \%$ of respondents indicated that use of aspirin may reduce the risk of stroke. For this item, gender was not associated with any differences in responses, however, older, better educated individuals and those living in the city listed more stroke symptoms than their counterparts (Table 5).

Adequate knowledge of stroke, as defined in the Methods section above, was assessed as a combination of three aspects: knowledge of major risk factors, recognition of most typical symptoms and a call to emergency services as a proper action. Risk factors and actions were recognized well by all participants (74.1\% and $82.6 \%$ participants, respectively), but knowledge of stroke symptoms was substantially lower $(47.4 \%)$, what was probably translated to low overall proportion of participants with adequate knowledge (36.2\%) (Table 6). Place of residence, education level, previous stroke in relatives or friends and to some degree gender, contributed to differences between subgroups in proportions of participants correctly recognizing stoke. Women, better educated individuals, those living in the city and who had previous contact with stroke know all critical aspects of stroke better than their counterparts.

In the univariate analysis, female gender $(\mathrm{OR}=0.606,95 \% \mathrm{CI}(0.465,0.790), p=0.001)$, living in the urban area $(\mathrm{OR}=1.757,95 \% \mathrm{CI}(1.350,2.287), p<0.001)$, better education level $(\mathrm{OR}=2.056,95 \% \mathrm{CI}(1.517$, $2.787), p<0.001)$, and previous occurrence of stroke in relatives or friends (OR $=2.998,95 \% \mathrm{CI}(2.298$, $3.910), p<0.001$ ) were statistically significant predictors of the adequate knowledge of stroke. However, age was not statistically significant predictor of the adequate knowledge of stroke, either considered as a continuous variable, or as a binary age groups ( $p=0.073$ and $p=0.134$, respectively). In the multivariate stepwise binary logistic regression, education duration, and previous occurrence of stroke in family or friends were identified as strongest independent predictors of adequate knowledge of stroke (both $p<0.001$ ) (Table 7). Gender and place of residence were also statistically significant predictors in the model, but age (in years) showed only a trend toward significance $(p=0.053)$. 
Table 4. Stroke symptoms provided by participants in response to an open-ended question grouped by gender, age group, place of residence, education level, and profession.

\begin{tabular}{|c|c|c|c|c|c|c|c|c|c|c|}
\hline & $\begin{array}{l}\text { Consciousness } \\
\text { Disturbances }\end{array}$ & Headache & $\begin{array}{l}\text { Speech } \\
\text { Disturbances }\end{array}$ & $\begin{array}{l}\text { Face or } \\
\text { mouth } \\
\text { Asymmetry }\end{array}$ & $\begin{array}{l}\text { Paresis, } \\
\text { Paralysis }\end{array}$ & $\begin{array}{l}\text { Sensory } \\
\text { Symptoms }\end{array}$ & $\begin{array}{l}\text { Vertigo, } \\
\text { Dizziness }\end{array}$ & $\begin{array}{l}\text { Nausea, } \\
\text { Vomiting }\end{array}$ & Other & Don't Know \\
\hline \multicolumn{11}{|l|}{ All } \\
\hline$n$ & 379 & 297 & 119 & 37 & 221 & 62 & 51 & 67 & 70 & 481 \\
\hline$\%$ & 33.4 & 26.2 & 10.5 & 3.3 & 19.5 & 5.5 & 4.5 & 5.9 & 6.2 & 42.4 \\
\hline \multicolumn{11}{|l|}{ Gender * } \\
\hline female, $n$ & 269 & 218 & 91 & 25 & 164 & 48 & 33 & 48 & 51 & 289 \\
\hline$\%$ & 35.8 & 29.0 & 12.1 & 3.3 & 21.8 & 6.4 & 4.4 & 6.4 & 6.8 & 38.4 \\
\hline male, $n$ & 110 & 79 & 28 & 12 & 57 & 14 & 18 & 19 & 19 & 192 \\
\hline$\%$ & 28.8 & 20.7 & 7.3 & 3.1 & 14.9 & 3.7 & 4.7 & 5.0 & 5.0 & 50.3 \\
\hline \multicolumn{11}{|l|}{ Age group } \\
\hline$<45$ years, $n$ & 218 & 195 & 74 & 16 & 150 & 39 & 35 & 42 & 46 & 297 \\
\hline$\%$ & 31.0 & 27.7 & 10.5 & 2.3 & 21.3 & 5.5 & 5.0 & 6.0 & 6.5 & 42.2 \\
\hline$\geq 45$ years, $n$ & 161 & 102 & 45 & 21 & 71 & 23 & 16 & 25 & 24 & 184 \\
\hline$\%$ & 37.4 & 23.7 & 10.4 & 4.9 & 16.5 & 5.3 & 3.7 & 5.8 & 5.6 & 42.7 \\
\hline \multicolumn{11}{|l|}{$\begin{array}{l}\text { Place of } \\
\text { residence * }\end{array}$} \\
\hline urban, $n$ & 261 & 185 & 88 & 27 & 152 & 50 & 32 & 47 & 56 & 296 \\
\hline$\%$ & 35.5 & 25.2 & 12.0 & 3.7 & 20.7 & 6.8 & 4.4 & 6.4 & 7.6 & 40.3 \\
\hline rural, $n$ & 118 & 112 & 31 & 10 & 69 & 12 & 19 & 20 & 14 & 185 \\
\hline$\%$ & 29.6 & 28.1 & 7.8 & 2.5 & 17.3 & 3.0 & 4.8 & 5.0 & 3.5 & 46.4 \\
\hline \multicolumn{11}{|l|}{$\begin{array}{l}\text { Education } \\
\text { duration * }\end{array}$} \\
\hline$<10$ years, $n$ & 75 & 56 & 13 & 11 & 31 & 12 & 12 & 15 & 6 & 152 \\
\hline$\%$ & 26.7 & 19.9 & 4.6 & 3.9 & 11.0 & 4.3 & 4.3 & 5.3 & 2.1 & 54.1 \\
\hline$>10$ years, $n$ & 304 & 241 & 106 & 26 & 190 & 50 & 39 & 52 & 64 & 329 \\
\hline$\%$ & 35.6 & 28.3 & 12.4 & 3.0 & 22.3 & 5.9 & 4.6 & 6.1 & 7.5 & 38.6 \\
\hline \multicolumn{11}{|c|}{ Stroke in relatives or friends * } \\
\hline yes, $n$ & 142 & 96 & 58 & 21 & 98 & 20 & 16 & 19 & 40 & 80 \\
\hline$\%$ & 43.4 & 29.4 & 17.7 & 6.4 & 30.0 & 6.1 & 4.9 & 5.8 & 12.2 & 24.5 \\
\hline no, $n$ & 237 & 201 & 61 & 16 & 123 & 42 & 35 & 48 & 30 & 401 \\
\hline$\%$ & 29.4 & 24.9 & 7.6 & 2.0 & 15.2 & 5.2 & 4.3 & 5.9 & 3.7 & 49.7 \\
\hline Profession & & & & & & & & & & \\
\hline
\end{tabular}


Table 4. Cont

\begin{tabular}{|c|c|c|c|c|c|c|c|c|c|c|}
\hline & $\begin{array}{l}\text { Consciousness } \\
\text { Disturbances }\end{array}$ & Headache & $\begin{array}{l}\text { Speech } \\
\text { Disturbances }\end{array}$ & $\begin{array}{l}\text { Face or } \\
\text { mouth } \\
\text { Asymmetry }\end{array}$ & $\begin{array}{l}\text { Paresis, } \\
\text { Paralysis }\end{array}$ & $\begin{array}{l}\text { Sensory } \\
\text { Symptoms }\end{array}$ & $\begin{array}{l}\text { Vertigo, } \\
\text { Dizziness }\end{array}$ & $\begin{array}{l}\text { Nausea, } \\
\text { Vomiting }\end{array}$ & Other & Don't Know \\
\hline $\begin{array}{l}\text { white-collar } \\
\text { worker, } n\end{array}$ & 154 & 111 & 61 & 15 & 107 & 29 & 20 & 26 & 32 & 180 \\
\hline$\%$ & 34.1 & 24.6 & 13.5 & 3.3 & 23.7 & 6.4 & 4.4 & 5.8 & 7.1 & 39.8 \\
\hline retired, $n$ & 91 & 70 & 24 & 7 & 38 & 16 & 9 & 11 & 9 & 126 \\
\hline$\%$ & 33.3 & 25.6 & 8.8 & 2.6 & 13.9 & 5.9 & 3.3 & 4.0 & 3.3 & 46.2 \\
\hline $\begin{array}{l}\text { manual worker, } \\
n\end{array}$ & 59 & 53 & 12 & 7 & 28 & 6 & 13 & 16 & 7 & 108 \\
\hline$\%$ & 26.2 & 23.6 & 5.3 & 3.1 & 12.4 & 2.7 & 5.8 & 7.1 & 3.1 & 48.0 \\
\hline unemployed, $n$ & 24 & 22 & 4 & 4 & 14 & 3 & 1 & 5 & 4 & 40 \\
\hline$\%$ & 31.2 & 28.6 & 5.2 & 5.2 & 18.2 & 3.9 & 1.3 & 6.5 & 5.2 & 51.9 \\
\hline $\begin{array}{l}\text { health care } \\
\text { professional, } n\end{array}$ & 38 & 31 & 15 & 4 & 29 & 7 & 8 & 8 & 14 & 12 \\
\hline$\%$ & 51.4 & 41.9 & 20.3 & 5.4 & 39.2 & 9.5 & 10.8 & 10.8 & 18.9 & 16.2 \\
\hline $\begin{array}{l}\text { private business, } \\
n\end{array}$ & 13 & 10 & 3 & 0 & 5 & 1 & 0 & 1 & 4 & 15 \\
\hline$\%$ & 39.4 & 30.3 & 9.1 & & 15.2 & 3.0 & & 3.0 & 12.1 & 45.5 \\
\hline
\end{tabular}

Note: Between-groups comparisons performed with Cochran-Mantel-Haenszel test, ${ }^{*}$ indicates $p<0.05$. Proportions in the Profession category were not analyzed. 
Table 5. Stroke prevention strategies indicated by participants in the response to close-ended question by gender, age group, place of residence, education level, and profession.

\begin{tabular}{|c|c|c|c|c|c|c|c|c|}
\hline & $\begin{array}{l}\text { Smoking } \\
\text { Cessation }\end{array}$ & $\begin{array}{l}\text { Moderate } \\
\text { Alcohol } \\
\text { Consumption }\end{array}$ & Low Fat Diet & Taking Aspirin & $\begin{array}{l}\text { Regular } \\
\text { Physical } \\
\text { Activity }\end{array}$ & $\begin{array}{l}\text { Healthy } \\
\text { Lifestyle }\end{array}$ & $\begin{array}{l}\text { Blood Pressure } \\
\text { Monitoring }\end{array}$ & $\begin{array}{l}\text { Taking } \\
\text { Vitamins }\end{array}$ \\
\hline \multicolumn{9}{|l|}{ All } \\
\hline$n$ & 680 & 367 & 559 & 265 & 656 & 877 & 771 & 155 \\
\hline$\%$ & 60.0 & 32.4 & 49.3 & 23.4 & 57.8 & 77.3 & 68.0 & 13.7 \\
\hline \multicolumn{9}{|l|}{ Gender } \\
\hline male, $n$ & 226 & 124 & 174 & 84 & 215 & 296 & 249 & 65 \\
\hline$\%$ & 59.2 & 32.5 & 45.5 & 22.0 & 56.3 & 77.5 & 65.2 & 17.0 \\
\hline female, $n$ & 454 & 243 & 385 & 181 & 441 & 581 & 522 & 90 \\
\hline$\%$ & 60.4 & 32.3 & 51.2 & 24.1 & 58.6 & 77.3 & 69.4 & 12.0 \\
\hline \multicolumn{9}{|l|}{ Age group * } \\
\hline$<45$ years, $n$ & 412 & 214 & 336 & 147 & 405 & 539 & 460 & 88 \\
\hline$\%$ & 58.6 & 30.4 & 47.8 & 20.9 & 57.6 & 76.7 & 65.4 & 12.5 \\
\hline$\geq 45$ years, $n$ & 268 & 153 & 223 & 118 & 251 & 338 & 311 & 67 \\
\hline$\%$ & 62.2 & 35.5 & 51.7 & 27.4 & 58.2 & 78.4 & 72.2 & 15.5 \\
\hline \multicolumn{9}{|l|}{$\begin{array}{l}\text { Place of } \\
\text { residence * }\end{array}$} \\
\hline urban, $n$ & 456 & 259 & 390 & 193 & 438 & 582 & 525 & 97 \\
\hline$\%$ & 62.0 & 35.2 & 53.1 & 26.3 & 59.6 & 79.2 & 71.4 & 13.2 \\
\hline rural, $n$ & 224 & 108 & 169 & 72 & 218 & 295 & 246 & 58 \\
\hline$\%$ & 56.1 & 27.1 & 42.4 & 18.0 & 54.6 & 73.9 & 61.7 & 14.5 \\
\hline \multicolumn{9}{|l|}{$\begin{array}{l}\text { Education } \\
\text { duration * }\end{array}$} \\
\hline$<10$ years, $n$ & 148 & 87 & 113 & 50 & 138 & 210 & 182 & 42 \\
\hline$\%$ & 52.7 & 31.0 & 40.2 & 17.8 & 49.1 & 74.7 & 64.8 & 14.9 \\
\hline$>10$ years, $n$ & 532 & 280 & 446 & 215 & 518 & 667 & 589 & 113 \\
\hline$\%$ & 62.4 & 32.8 & 52.3 & 25.2 & 60.7 & 78.2 & 69.1 & 13.2 \\
\hline \multicolumn{9}{|l|}{$\begin{array}{l}\text { Stroke in } \\
\text { relatives or } \\
\text { friends * }\end{array}$} \\
\hline yes, $n$ & 215 & 121 & 194 & 92 & 219 & 261 & 246 & 37 \\
\hline$\%$ & 65.7 & 37.0 & 59.3 & 28.1 & 67.0 & 79.8 & 75.2 & 11.3 \\
\hline no, $n$ & 465 & 246 & 365 & 173 & 437 & 617 & 525 & 118 \\
\hline$\%$ & 57.6 & 30.5 & 45.2 & 21.4 & 54.2 & 76.5 & 65.1 & 14.6 \\
\hline
\end{tabular}


Table 5. Cont.

\begin{tabular}{|c|c|c|c|c|c|c|c|c|}
\hline & $\begin{array}{l}\text { Smoking } \\
\text { Cessation }\end{array}$ & $\begin{array}{l}\text { Moderate } \\
\text { Alcohol } \\
\text { Consumption }\end{array}$ & Low Fat Diet & Taking Aspirin & $\begin{array}{l}\text { Regular } \\
\text { Physical } \\
\text { Activity }\end{array}$ & $\begin{array}{l}\text { Healthy } \\
\text { Lifestyle }\end{array}$ & $\begin{array}{l}\text { Blood Pressure } \\
\text { Monitoring }\end{array}$ & $\begin{array}{l}\text { Taking } \\
\text { Vitamins }\end{array}$ \\
\hline $\begin{array}{l}\text { Profession } \\
\text { white-collar } \\
\text { worker, } n\end{array}$ & 277 & 148 & 234 & 99 & 282 & 361 & 300 & 56 \\
\hline$\%$ & 61.3 & 32.7 & 51.8 & 21.9 & 62.4 & 79.9 & 66.4 & 12.4 \\
\hline retired, $n$ & 157 & 90 & 130 & 76 & 146 & 204 & 193 & 37 \\
\hline$\%$ & 57.5 & 33.0 & 47.6 & 27.8 & 53.5 & 74.7 & 70.7 & 13.6 \\
\hline $\begin{array}{l}\text { manual worker, } \\
n\end{array}$ & 125 & 69 & 88 & 37 & 117 & 166 & 146 & 42 \\
\hline$\%$ & 55.6 & 30.7 & 39.1 & 16.4 & 52.0 & 73.8 & 64.9 & 18.7 \\
\hline unemployed, $n$ & 48 & 20 & 36 & 12 & 37 & 58 & 49 & 10 \\
\hline$\%$ & 62.3 & 26.0 & 46.8 & 15.6 & 48.1 & 75.3 & 63.6 & 13.0 \\
\hline $\begin{array}{l}\text { health care } \\
\text { professional, } n\end{array}$ & 55 & 28 & 52 & 28 & 52 & 61 & 59 & 7 \\
\hline$\%$ & 74.3 & 37.8 & 70.3 & 37.8 & 70.3 & 82.4 & 79.7 & 9.5 \\
\hline $\begin{array}{l}\text { private business, } \\
n\end{array}$ & 18 & 12 & 19 & 13 & 22 & 27 & 24 & 3 \\
\hline$\%$ & 54.5 & 36.4 & 57.6 & 39.4 & 66.7 & 81.8 & 72.7 & 9.1 \\
\hline
\end{tabular}

Note: Between-groups comparisons performed with Cochran Mantel Haenszel test, ${ }^{*}$ indicates $p<0.05$. Proportions in the Profession category were not analyzed. 
Table 6. Adequate knowledge of stroke as a combined measure of correct knowledge of risk factors, symptoms and necessary actions by gender, age group, place of residence, education level, and profession.

\begin{tabular}{|c|c|c|c|c|c|c|c|c|}
\hline \multirow{2}{*}{$\begin{array}{l}\text { n, \% } \\
\text { All }\end{array}$} & \multicolumn{2}{|c|}{$\begin{array}{l}\text { Knows at Least One of Risk } \\
\text { Factors }\end{array}$} & \multicolumn{2}{|c|}{$\begin{array}{l}\text { Names Correctly at Least One of } \\
\text { Typical Signs or Symptoms }\end{array}$} & \multicolumn{2}{|c|}{$\begin{array}{l}\text { Correct Action Taken (Call } \\
\text { Emergency) }\end{array}$} & \multicolumn{2}{|c|}{$\begin{array}{l}\text { Adequate Knowledge (Correctly } \\
\text { Identified All Three Conditions) }\end{array}$} \\
\hline & 840 & $74.1 \%$ & 537 & $47.4 \%$ & 937 & $82.6 \%$ & 411 & $36.2 \%$ \\
\hline Gender & & & & & & & & \\
\hline female & 564 & $75.0 \%$ & 385 & $51.2 \%$ & 633 & $84.2 \%$ & 301 & $40.0 \%$ \\
\hline male & 276 & $72.3 \%$ & 152 & $39.8 \%$ & 304 & $79.6 \%$ & 110 & $28.8 \%$ \\
\hline \multicolumn{9}{|l|}{ Age group } \\
\hline$<45$ years & 510 & $72.5 \%$ & 325 & $46.2 \%$ & 578 & $82.2 \%$ & 243 & $34.6 \%$ \\
\hline$\geq 45$ years & 330 & $76.6 \%$ & 212 & $49.2 \%$ & 359 & $83.3 \%$ & 168 & $39.0 \%$ \\
\hline \multicolumn{9}{|l|}{$\begin{array}{l}\text { Place of } \\
\text { residence }\end{array}$} \\
\hline urban & 563 & $76.6 \%$ & 376 & $51.2 \%$ & 618 & $84.1 \%$ & 299 & $40.7 \%$ \\
\hline rural & 277 & $69.4 \%$ & 161 & $40.4 \%$ & 319 & $79.9 \%$ & 112 & $28.1 \%$ \\
\hline \multicolumn{9}{|l|}{$\begin{array}{l}\text { Education } \\
\text { duration }\end{array}$} \\
\hline$<10$ years & 187 & $66.5 \%$ & 100 & $35.6 \%$ & 219 & $77.9 \%$ & 69 & $24.6 \%$ \\
\hline$\geq 10$ years & 653 & $76.6 \%$ & 437 & $51.2 \%$ & 718 & $84.2 \%$ & 342 & $40.1 \%$ \\
\hline \multicolumn{9}{|c|}{ Stroke in relatives or friends } \\
\hline yes & 284 & $86.8 \%$ & 213 & $65.1 \%$ & 293 & $89.6 \%$ & 179 & $54.7 \%$ \\
\hline no & 556 & $68.9 \%$ & 324 & $40.1 \%$ & 644 & $79.8 \%$ & 232 & $28.7 \%$ \\
\hline \multicolumn{9}{|l|}{ Profession } \\
\hline $\begin{array}{l}\text { white-collar } \\
\text { worker }\end{array}$ & 345 & $76.3 \%$ & 231 & $51.1 \%$ & 391 & $86.5 \%$ & 186 & $41.2 \%$ \\
\hline retired & 194 & $71.1 \%$ & 122 & $44.7 \%$ & 223 & $81.7 \%$ & 91 & $33.3 \%$ \\
\hline manual worker & 157 & $69.8 \%$ & 84 & $37.3 \%$ & 170 & $75.6 \%$ & 53 & $23.6 \%$ \\
\hline unemployed & 53 & $68.8 \%$ & 29 & $37.7 \%$ & 62 & $80.5 \%$ & 20 & $26.0 \%$ \\
\hline $\begin{array}{l}\text { health care } \\
\text { professional }\end{array}$ & 67 & $90.5 \%$ & 55 & $74.3 \%$ & 62 & $83.8 \%$ & 46 & $62.2 \%$ \\
\hline private business & 24 & $72.7 \%$ & 16 & $48.5 \%$ & 29 & $87.9 \%$ & 15 & $45.5 \%$ \\
\hline
\end{tabular}

Note: Between-groups comparisons performed with chi-squared test, bold indicates $p<0.05$. Proportions in the Profession category were not analyzed. 
Table 7. Multivariate stepwise binary logistic regression model to identify independent predictors of adequate knowledge of stroke.

\begin{tabular}{llccc}
\hline Parameter & Values & Estimate & OR (95\% CI) & $p$-Value \\
\hline Gender & Female $=0$, male $=1$ & -0.362 & $0.696(0.526,0.921)$ & 0.011 \\
Age & years & 0.009 & $1.009(1.000,1.018)$ & 0.053 \\
Place of residence & Rural = 0, urban =1 & 0.358 & $1.430(1.077,1.899)$ & 0.013 \\
Education duration & $<10$ years = 0, $\geq 10$ years $=1$ & 0.688 & $1.989(1.432,2.763)$ & $<0.001$ \\
Stroke in family or & No = 0, yes = & 1.039 & $2.826(2.153,3.710)$ & $<0.001$ \\
friends & & -1.914 & & $<0.001$ \\
Constant & & & \\
\hline
\end{tabular}

Note: $p$-value of the model $<0.001$, McFadden's Rho $^{2}=0.072$.

\section{Discussion}

Identifying the main risk factors for cerebrovascular disease and the knowledge of its major symptoms directly translates into stroke prevention and medical intervention, including thrombolysis. Our research regarding public awareness of stroke is the first in Poland carried out in such a large population. The work is based on a survey, mainly without the suggestion of an answer, which is a big value regarding already well-established knowledge and the way in which respondents reply. The advantage of the survey was the form of open questions, also used in several other publications $[5,8]$.

In our study, 834 respondents chose a correct stroke definition (73.5\%). In the study by Neau et al. [22], the majority of respondents $(76.9 \%)$ indicated the brain as an organ affected by stroke. Similar results (85\%) were obtained by Mikulik et al. [23] but a knowledge of definition of stroke is not the same as awareness of stroke symptoms and risk factor, so it is definitely not enough to prevent stroke or give an appropriate reaction to a hypothetical case of stroke. The vast majority of our respondents indicated that stroke is an emergency and requires hospitalization $(92.8 \%$ and $97.5 \%$, respectively). There were $82.6 \%$ of respondents who would call an emergency if stroke was suspected, while $13.8 \%$ would see their GP, and only $6 \%$ would wait for the symptoms to resolve. As shown by Joes et al. [3], published data is divergent in various publications, with $27 \%$ up to $100 \%$ of respondents declaring that they would call an ambulance in the first place if stroke is suspected, $3 \%$ to $40 \%$ who would see their GP, and $1 \%$ to $10 \%$ who would not take any action.

In our study $57.6 \%$ of the participants were able to list at least one symptom, while $35.2 \%$ respondents listed two or more symptoms. Respondents were asked to list all known symptoms of stroke. The women, better educated individuals, those living in the city and who had previous contact with stroke listed more symptoms than their counterparts. The ability to name one symptom of stroke differed significantly between studies ranging between $25 \%$ and $100 \%$ [3]. Just as in the case of the risk factors, stroke symptoms were less recognized if open-ended questions were used. In one study, only $38 \%$ of participants were able to identify one or more symptoms when open-ended questions were asked, compared to $100 \%$ when a list of possible symptoms was shown [24]. In the largest European study (11.827 Spanish adults), Lundelin et al. (2012) reported that over 11\% individuals could not list any symptom of stroke and one in five people indicated that they would do something other than call an ambulance if they think that someone has a stroke [6]. In our study $42.4 \%$ of the respondents haven't known any stroke symptoms and the most frequently listed are: consciousness disturbances $(33.4 \%)$ and headache $(26.2 \%)$. Muscle weakness was reported by $19.5 \%$ and speech disturbances by $10.6 \%$ of the individuals. In other studies, the respondents most often mentioned paresis, speech disorders and dizziness [22,25-27]. On the other hand, vision impairment, diplopia, and gait disturbance were mentioned rare $(<5 \%)$, in the presented study not once. Masztalewicz at al. studied the percentage of callers (potentially every respondent in our study) who recognized stroke symptoms in the Polish West Pomerania. In similar period of time (2013-2014) correct answer listed 39.9\% individuals, who called emergency because noted stroke symptoms [18]. 
In the publication Teuschl et al. (2010) regarding the impact of the awareness of stroke factors on delayed transport to the hospital highlighted the weak impact of education and sociodemographic data, such as age or residential area on the reaction taken after the onset of symptoms of stroke, and only $25-56 \%$ of patients recognized symptoms of stroke [4]. The disadvantages of the survey was to list disturbances of consciousness and headaches among symptoms associated with stroke, because the symptoms are not typical of acute ischemic stroke.

Awareness of stroke symptoms was better but not perfect among Emergency Medical Services (EMS) employees in Dubai: 79\% of EMS staff were able to name the symptoms of a stroke [5].

In our study $24.2 \%$ of the respondents did not name any stroke risk factor, $39 \%$ were able to name at least one of them, $20 \%$ named two risk factors, and $10 \%$ were able to name three or more. In the study by Gongor-Rivera et al. [28] it was 33.3\% (none), 29.4\% (at least 1 factor), 25.2\% (two or more factors), and only $12.1 \%$ (three or more factors), respectively. The proportion of individuals naming one or more stroke risk factors differed significantly between studies ranging between $18 \%$ and $94 \%$ for open-ended questions and between $42 \%$ and $97 \%$ for closed-ended questions [3]. In a large urban population in France, the majority of patients $(62.3 \%)$ were able to name three risk factors for stroke, but omitted the most important of them, and less than half of the respondents were able to recognize one or more warning signs. They based their knowledge on magazines and books, as well as on the experience of families in whom stroke occurred [22]. In our study not press but television was the main source of information of stroke.

The stroke risk factors mentioned most often by our respondents were an old age $(55.3 \%$, but it was separate question in opposite to the cited below), then hypertension $(29.2 \%)$, smoking $(10.8 \%)$, cardiovascular disease $(8.6 \%)$, and stress $(13.1 \%)$. Lipid disorders, diabetes, obesity, and genetic factors were mentioned by less than $5 \%$ of all respondents. In other studies, hypertension and smoking were the most frequently mentioned risk factors for stroke, whereas diabetes, obesity, heart disease (including cardiomyopathy and atrial fibrillation), using oral contraceptives, and old age were mentioned by less than $5 \%$ of individuals [1,22-24]. In our study, in the closed-ended question on stroke prevention strategies, a healthy lifestyle and regular blood pressure control were chosen as the best prevention ( $77 \%$ and $68 \%$, respectively). $60 \%$ of our respondents also indicated smoking cessation and $58 \%$ regular physical activity.

In Table 8 some data regarding stroke awareness received from different population were compared. The methods used in these study, cultural conditions and interpretation of results are different but we can say that in Poland the awareness of stroke is similar as in the rest of the world, but not sufficient. The only significant difference seems to be the symptom of stroke most frequently reported by the respondents. However, such differences are not visible in the case of the most frequently mentioned risk factors of stroke.

Our arbitrary definition of "adequate stroke knowledge" is based on Ramirez-Moreno et al. and Segura et al., but a little bit more conservative (just five main typical stroke symptoms) $[20,21]$. In the first mentioned paper, $40 \%$ of the respondents have adequate stroke knowledge, which is comparable to our study (36.2\%). We also confirmed, that poorly educated people have less knowledge, but not older people. So the situation is quite similar in Poland and in Spain [20]. "I call an emergency" was answered by $82.6 \%$ in our group, but $24.2 \%$ of the people did not list one risk factor, and $42.4 \%$ did not know one stroke symptom. According the authors of this study such a general stroke awareness is not sufficient to improve a rate of thrombolysis or to favor the healthy behaviors. Because the most respondents (in our study 58.7\%, in Ramirez-Moreno at al. study 53.6\%) named TV as an source of stroke information, it means that TV campaign or advertisements are not focused on proper issues and should be more informative. According to our results" stroke in relatives or friends' is the strongest independent predictors for adequate stroke knowledge, therefore media should be encouraged to provide sufficient and more detailed information focused on virtual stroke presentation. This may help to shape education campaigns referring to close relatives, or even provide virtual patient for the 
purpose of personification of stroke victim to be more impactful and emotional. It is known, that the information processed during emotional situations is remembered for very long time.

Early recognition of stroke symptoms is the key to maximizing the opportunity for medical intervention, in particular thrombolysis. Our study highlights some gaps in common knowledge of strokes. We need to improve public awareness of stroke in Poland. In our study only $13 \%$ of the respondents heard something about stroke whereas social campaign on cervical cancer prevention reached $79 \%$ of them. Currently, campaigns are in progress in Poland under the auspices of the Polish Neurological Society (stop strokes, prevent.pl, time is important, and open hand after stroke), the aim of which is to spread awareness of stroke prevention and diagnosis and post-stroke assistance. Public campaigns, as shown in research [30,31,35], are among the best ways to achieve this. In the Polish papers, Kobayashi et al. (2018) and Masztalewicz et al. (2016) emphasizes that despite very low quality of evidence, educational campaigns to increase the awareness of immediately calling emergency medical services are strongly recommended $[7,18]$.

The presented study has some limitations. First, the disadvantage of the work was the construction of the questionnaire which ignored time as a risk factor for the consolidation of neurological symptoms. Current ongoing campaigns are focused on the time factor in the development of stroke symptoms and a key factor in the management of acute stroke, in particular thrombolysis. Second, using the "don't know" option in open-ended questions may have caused more respondents to choose it without thinking about the right answer. Thus, a large number of undecided subjects may not be indicative of a failure to recognize stroke symptoms, but may be caused by the lack of willingness to fill in the questionnaire reliably. Perhaps, therefore, the "don't know" option should have been removed from the questionnaire, which would have prompted a greater number of respondents to provide their own answers to open-ended questions. 
Table 8. Some data regarding stroke awareness received from different population.

\begin{tabular}{|c|c|c|c|c|c|}
\hline & $\begin{array}{l}\% \text { of Subjects Reported } \\
\text { at Least } 1 \text { Correct } \\
\text { Warning Sign of Stroke }\end{array}$ & $\begin{array}{l}\text { The Most Frequent } \\
\text { Mentioned Warning } \\
\text { Sign of Stroke }\end{array}$ & $\begin{array}{l}\% \text { of Subjects Named at } \\
\text { Least } 1 \text { Correct Risk } \\
\text { Factor for Stroke }\end{array}$ & $\begin{array}{l}\text { The Most Frequent } \\
\text { Mentioned Risk Factor } \\
\text { for Stroke }\end{array}$ & $\begin{array}{l}\% \text { of Subjects Calls } \\
\text { an Emergency }\end{array}$ \\
\hline $\begin{array}{l}\text { Krzystanek et al. } \\
\text { (Poland) }\end{array}$ & 57.6 & $\begin{array}{c}\text { consciousness } \\
\text { disturbances }\end{array}$ & 76.8 & $\begin{array}{c}\text { old age, } \\
\text { high blood pressure }\end{array}$ & 82.6 \\
\hline $\begin{array}{l}\text { Ramirez-Moreno et al. } \\
\quad(\text { Spain, 2015) [20] }\end{array}$ & 73.0 & paralysis/weakness & 59.2 & $\begin{array}{l}\text { smoking, high blood } \\
\text { pressure }\end{array}$ & 80 \\
\hline $\begin{array}{c}\text { Hickey et al. } \\
\text { (Ireland, 2009) [29] }\end{array}$ & 87.0 & slurred speech & 94.0 & hypertension & no data \\
\hline $\begin{array}{c}\backslash \text { Metias et al. } \\
\text { (Canada, 2017) [30] }\end{array}$ & 88.7 & $\begin{array}{l}\text { weakness, numbness or } \\
\text { paralysis }\end{array}$ & 83.1 & $\begin{array}{l}\text { smoking, high blood } \\
\text { pressure }\end{array}$ & 94.1 \\
\hline $\begin{array}{l}\text { Nordanstig et al. } \\
\text { (Sweden, 2017) [31] }\end{array}$ & 72.0 & speech disturbance & no data & smoking, stress & 65.0 \\
\hline $\begin{array}{c}\text { Akiyama et al. } \\
\text { (Japan, 2013) [32] }\end{array}$ & no data & speech disturbances & no data & $\begin{array}{c}\text { high blood pressure, } \\
\text { diabetes }\end{array}$ & 67.0 \\
\hline $\begin{array}{l}\text { Krishnamurthi et al. } \\
\text { (New Zealand, 2020) [33] }\end{array}$ & 98.0 & $\begin{array}{c}\text { sudden difficulty } \\
\text { speaking or } \\
\text { understanding others }\end{array}$ & 78.0 & high blood pressure & 80.0 \\
\hline $\begin{array}{l}\text { Jackson et al. } \\
(\text { USA, 2020) [34] }\end{array}$ & no data & $\begin{array}{c}\text { face, arm, leg, side } \\
\text { numbness }\end{array}$ & no data & no data & 96.3 \\
\hline
\end{tabular}




\section{Conclusions}

1. Knowledge of stroke in the population of southern Poland is low and may be considered insufficient to address the needs of timely management in the action chain. 2. Previous occurrence of stroke in the relatives or friends is the most impacting factor for adequate knowledge of stroke.

Author Contributions: Conceptualization, E.K., M.Ś., and J.G.; methodology, E.K., J.G., and W.G.; formal analysis, E.K., M.Ś., and A.K.-K.; investigation, E.K., A.K.-K., and M.Ś.; resources, E.K., M.Ś., and J.G.; data curation, E.K., M.Ś, J.G., and A.K.-K.; writing-original draft preparation, E.K., J.G., and A.K.-K.; writing-review and editing, E.K., A.K.-K., and W.G.; supervision, E.K. and J.G.; project administration, E.K. and M.S. All authors have read and agreed to the published version of the manuscript.

Funding: This research received no external funding.

Acknowledgments: The authors gratefully acknowledge the assistance of Karolina Kalisz in proofreading the manuscript and Malwina Szynkaruk and Katarzyna Oleksiak in data collection.

Conflicts of Interest: The authors declare no conflict of interest.

\section{References}

1. Dearborn, J.L.; McCullough, L.D. Perception of risk and knowledge of risk factors in women at high risk for stroke. Stroke 2009, 40, 1181-1186. [CrossRef] [PubMed]

2. Banecka-Majkutewicz, Z.; Dobkowska, M.; Wichowicz, H. Analiza czynników ryzyka udaru niedokrwiennego mózgu. Ann. Acad. Med. Gedan. 2005, 35, 207-216.

3. Jones, S.P.; Jenkinson, A.J.; Leathley, M.J.; Watkins, C.L. Stroke knowledge and awareness: An integrative review of the evidence. Age Ageing 2010, 39, 11-22. [CrossRef]

4. TeuschI, Y.; Brainin, M. Stroke education: Discrepancies among factors influencing prehospital delay and stroke knowledge. Int. J. Stroke 2010, 5, 187-208. [CrossRef] [PubMed]

5. Shire, F.; Kasim, Z.; Alrukn, S.; Khan, M. Stroke awareness among Dubai emergency medical service staff and impact of an educational intervention. BMC Res. Notes 2017, 10,1-6. [CrossRef]

6. Lundelin, K.; Graciani, A.; García-Puig, J.; Guallar-Castillón, P.; Taboada, J.M.; Rodríguez-Artalejo, F.; Banegas, J.R. Knowledge of stroke warning symptoms and intended action in response to stroke in Spain: A nationwide population-based study. Cerebrovasc. Dis. 2012, 34, 161-168. [CrossRef] [PubMed]

7. Kobayashi, A.; Czlonkowska, A.; Ford, G.A.; Fonseca, A.C.; Luijckx, G.J.; Korv, J.; De La Ossa, N.P.; Price, C.I.; Russell, D.; Tsiskaridze, A.; et al. European Academy of Neurology and European Stroke Organization consensus statement and practical guidance for pre-hospital management of stroke. Eur. J. Neurol. 2018, 25, 425-433. [CrossRef] [PubMed]

8. Kawecka-Jaszcz, K.; Rajzer, M. Pacjent z nadciśnieniem tętniczym po udarze mózgu. Indywidualizacja. Terapia nadciśnienia tętniczego. Folia Cardiol. Ex. 2007, 2, 335-342.

9. Klimaszewska, K.; Krajewska-Kułak, E.; Jankowiak, B.; Rolka, H.; Kowalczuk, K. Charakterystyka usprawniania chorych na udar mózgu w poszczególnych okresach rehabilitacji. Ann. Acad. Med. Siles 2006, $60,41-46$.

10. Powers, W.J.; Rabinstein, A.A.; Ackerson, T.; Adeoye, O.M.; Bambakidis, N.C.; Becker, K.; Biller, J.; Brown, M.; Demaerschalk, B.M.; Hoh, B.; et al. 2018 Guidelines for the Early Management of Patients with Acute Ischemic Stroke: A Guideline for Healthcare Professionals from the American Heart Association/American Stroke Association. Stroke 2018, 49, e46-e99. [PubMed]

11. California Acute Stroke Pilot Registry (CASPR) Investigators. Prioritizing Interventions to Improve Rates of Thrombolysis for Ischemic Stroke. Neurology 2005, 64, 654-659. [CrossRef] [PubMed]

12. Yu, R.F.; Jose, M.C.Z.S.; Manzanilla, B.M.; Oris, M.Y.; Gan, R. Sources and reasons for delays in the care of acute stroke patients. J. Neurol. Sci. 2002, 199, 49-54. [CrossRef]

13. Engelstein, E.; Margulies, J.; Jeret, J.S. Lack of t-PA use for acute ischemic stroke in a community hospital: High incidence of exclusion criteria. Am. J. Emerg. Med. 2000, 18, 257-260. [CrossRef]

14. Kothari, R.; Sauerbeck, L.; Jauch, E.C.; Broderick, J.P.; Brott, T.; Khoury, J.; Liu, T. Patients' awareness of stroke signs, symptoms, and risk factors. Stroke 1997, 28, 1871-1875. [CrossRef] [PubMed] 
15. Schneider, A.T.; Pancioli, A.M.; Khoury, J.C.; Rademacher, E.; Tuchfarber, A.; Miller, R.; Woo, D.; Kissela, B.; Broderick, J.P. Trends in community knowledge of the warning signs and risk factors for stroke. JAMA 2003, 289, 343-346. [CrossRef] [PubMed]

16. Centers for Disease Control and Prevention (CDC). Awareness of stroke warning signs-17 states and the US Virgin Islands, 2001. Morb. Mortal. Wkly. Rep. 2004, 53, 359-362.

17. Yoon, S.S.; Heller, R.F.; Levi, C.; Wiggers, J.; Fitzgerald, P.E. Knowledge of stroke risk factors, warning symptoms, and treatment among an australian urban population. Stroke 2001, 32, 1926-1930. [CrossRef]

18. Masztalewicz, M.; Nowacki, P.; Kotlęga, D.; Czajkowska, A.B. Early Emergency Medical Service Calls for Stroke: Was the Long-Term Education Program Based on the Experience of West Pomerania Successful? J. Stroke Cerebrovasc. Dis. 2016, 25, 254-258. [CrossRef]

19. Procesy i struktury demograficzno-społeczne na obszarze województwa śląskiego w latach 1988-2008. Urząd Statystyczny w Katowicach, November 2010. Available online: https://katowice.stat.gov.pl/ download/gfx/katowice/pl/defaultaktualnosci/756/5/1/7/procesy_i_str_demograf1998-2008.pdf (accessed on 15 December 2020).

20. Ramirez-Moreno, J.M.; Alonso-Gonzalez, R.; Peral-Pacheco, D.; Millán-Núñez, M.V.; Aguirre-Sánchez, J.J. Stroke awareness is worse among the old and poorly educated: A population-based survey. J. Stroke Cerebrovasc. Dis. 2015, 24, 1038-1046. [CrossRef]

21. Segura, T.; Vega, G.; López, S.; Rubio, F.; Castillo, J. Public perception of stroke in Spain. J. Cerebrovasc. Dis. 2003, 16, 21-26. [CrossRef]

22. Neau, J.-P.; Ingrand, P.; Godeneche, G. Awareness within the French population concerning stroke signs, symptoms, and risk factors. Clin. Neurol. Neurosurg. 2009, 111, 659-664. [CrossRef]

23. Mikulík, R.; Bunt, L.; Hrdlicka, D.; Dusek, L.; Václavík, D.; Kryza, J. Calling 911 in response to stroke. A nationwide study assessing defini-tive individual behavior. Stroke 2008, 39, 1844-1849. [CrossRef] [PubMed]

24. Pontes-Neto, O.M.; Silva, G.S.; Feitosa, M.R.; de Figueiredo, N.L.; Fiorot, J.A., Jr.; Rocha, T.N.; Massaro, A.R.; Leite, J.P. Stroke awareness in Brazil. Alarming results in community-based study. Stroke 2008, 39, 292-296. [CrossRef]

25. De Dominicis, L.; Cardinali, P.; Pucci, E.; Marchegiani, G.; Caporalini, R.; Moretti, V.; Sanguigni, S.; Carle, F.; Gesuita, R.; Giuliani, G. What do Italians at high risk of stroke know about ischaemic stroke? A survey among a group of subjects undergoing neuro-sonographic examination. Neurol. Sci. 2006, 27, 7-13. [CrossRef] [PubMed]

26. Müller-Nordhorn, J.; Nolte, C.H.; Rossnagel, K.; Jungehülsing, G.J.; Reich, A.; Roll, S.; Villringer, A.; Willich, S.N. Knowledge about risk factors for stroke: A population-based survey with 28,090 participants. Stroke 2006, 37, 946-950. [CrossRef] [PubMed]

27. Campos-Sousa, R.N.; Soares, V.Y.R.; Almeida, K.J.S.; De Carvalho, L.I.M.; Jacobina, K.S.; Netto, A.E.A.; Macêdo, E.D.A.; Veloso, L.A. Knowledge of stroke among a Brazilian urban population. Arq. de Neuro-Psiquiatria 2007, 65, 587-591. [CrossRef] [PubMed]

28. Góngora-Rivera, F.; Gutiérrez-Jiménez, E.; Zenteno, M. Knowledge of ischemic stroke among a Mexico City population. J. Stroke Cerebrovasc. Dis. 2009, 18, 208-213. [CrossRef] [PubMed]

29. Hickey, A.; O'Hanlon, A.; McGee, H.; Donnellan, C.; Shelley, E.B.; Horgan, F.; O'Neill, D. Stroke awareness in the general population: Knowledge of stroke risk factors and warning signs in older adults. BMC Geriatr. 2009, 9, 35. [CrossRef]

30. Cruz, V.T.; Araujo, I.; Alves, I.; Magano, A.; Coutinho, P. Freeze the Stroke Public Awareness Program for Immediate Detection of First Symptoms. Stroke 2012, 43, 2510-2512. [CrossRef]

31. Metias, M.M.; Eisenberg, N.; Clemente, M.D.; Wooster, E.M.; Dueck, A.D.; Wooster, D.L.; Roche-Nagle, G. Public health campaigns and their effect on stroke knowledge in a high-risk urban population: A five-year study. Vascular 2017, 25, 497-503. [CrossRef]

32. Nordanstig, A.; Asplund, K.; Norrving, B.; Wahlgren, N.; Wester, P.; Rosengren, L. Impact of the Swedish National Stroke Campaign on stroke awareness. Acta Neurol. Scand. 2017, 136, 345-351. [CrossRef] [PubMed]

33. Akiyama, H.; Hasegawa, Y. Stroke Knowledge: A Nationwide, Internet-based Survey of 11,121 Inhabit-ants in Japan. Intern. Med. 2013, 52, 529-537. [CrossRef] [PubMed] 
34. Krishnamurthi, R.V.; Barker-Collo, S.; Barber, P.A.; Tippett, L.J.; Dalrymple-Alford, J.C.; Tunnage, B.; Mahon, S.; Parmar, P.G.; Moylan, M.; Feigin, V.L. Community Knowledge and Awareness of Stroke in New Zealand. J. Stroke Cerebrovasc. Dis. 2020, 29, 104589. [CrossRef] [PubMed]

35. Jackson, S.L.; Legvold, B.; Vahratian, A.; Blackwell, D.L.; Fang, J.; Gillespie, C.; Hayes, D.; Loustalot, F. Sociodemographic and Geographic Variation in Awareness of Stroke Signs and Symptoms among Adults-United States, 2017. Morb. Mortal. Wkly. Rep. 2020, 69, 1617-1621. [CrossRef]

Publisher's Note: MDPI stays neutral with regard to jurisdictional claims in published maps and institutional affiliations.

(C) 2020 by the authors. Licensee MDPI, Basel, Switzerland. This article is an open access article distributed under the terms and conditions of the Creative Commons Attribution (CC BY) license (http://creativecommons.org/licenses/by/4.0/). 